\title{
Influence of thermal treatment on thermal properties of adamantane derivatives
}

\author{
D. Szewczyk and A. Jeżowski \\ W. Trzebiatowski Institute of Low Temperatures and Structure Research Polish Academy of Sciences \\ Okólna 2, Wrocław 50-422, Poland
}

A.I. Krivchikov

B. Verkin Institute for Low Temperature Physics and Engineering of the National Academy of Sciences of Ukraine 47 Lenin Ave., Kharkov 61103, Ukraine

E-mail: krivchikov@ilt.kharkov.ua

\section{J.LI. Tamarit}

Grup de Caracterització de Materials, Departament de Física i Enginyieria Nuclear, ETSEIB, Universitat Politècnica de Catalunya, Diagonal 647, Barcelona 08028, Catalonia, Spain

Received February 3, 2015, published online April 23, 2015

\begin{abstract}
Heat transport mechanisms present in 2-adamantanone and 1-cyanoadamantane crystals were investigated in a broad temperature range. To characterize scattering processes, thermal conductivity and heat capacity measurements were carried out. A particular care was paid to the cooling rate of specimen which influenced the thermal history of the samples. The experimental results led to a conclusion that under slow cooling the thermal conductivity reaches the highest values and resembles the behavior of ordered molecular crystals. As for fast cooling, the "quenching" resulted in changes in both the structure and the temperature dependence of the thermal conductivity, the latter resembling that of amorphous solids. In heat capacity measurements the thermal history made on samples did not reflect the preliminary findings known from thermal conductivity results, which could imply that the observed mechanisms are more complex.
\end{abstract}

PACS: 44.35.+c Heat flow in multiphase systems;

61.43.-j Disorder solids;

65.60.+a Thermal properties of amorphous solids and glasses: heat capacity, thermal expansion, etc.

Keywords: thermal conductivity, phase transition, thermal treatment, molecular disorder, glassy dynamics.

\section{Introduction}

Plastic crystals have been already recognized [1] as crystals that exhibit unique properties intermediate between the systems in which the long-range positional and orientational order/disorder exists. In this kind of materials the molecules are dynamically disordered while their centers of mass form a regular lattice with respect to their orientational degrees of freedom. Most of the plastic crystals consist of molecules with close to globular shape, behaving like spherical entities rotating about molecular axes [2]. Because these materials show many features of the glassy dynamics they could serve as model systems for amorphous solids. One of the reasons for studying the glassy state behavior in plastic crystals is that these systems retain translational symmetry, and this property could provide insight into the metastability of molecular systems. Let us consider a glassy crystal, which is obtained by sufficiently fast cooling from an orientationally disordered phase (the so-called plastic phase). By this kind of thermal treatment the high-temperature dynamically disordered state can be supercooled, thereby avoiding a complete orientational order typical of low-temperature phases. As a result, an orientationally disordered state is reached below the glass transition temperature. Actually, the dynamics of the orientational relaxation in plastic crystals resembles in many respects that in conventional glass formers [3].

One of the best known plastic crystals is adamantane $\mathrm{C}_{10} \mathrm{H}_{16}$. This material has become a parent molecule for a large number of derivatives, obtained by substituting one 
or two hydrogen atoms. Unsubstituted adamantane shows a series thermally induced solid-to-solid phase transitions below melting. Another characteristic feature is that it shows deviations from the exponential relaxation and a non-Arrhenius behavior. Up to now, for many of adamantane derivatives, like 2-adamantanone, 1-cyanoadamantane, 1-chloroadamantane, a glassy freezing has been reported [4-6]. All those materials have been already studied by a wide variety of experimental methods, but for the first time we will focus on the thermal transport mechanisms. We believe that thermal conductivity measurements and heat capacity (in an expanded temperature range) may provide a better insight into thermal behavior of these disordered molecular systems. For our measurements we have chosen 2-adamantanone, $\mathrm{C}_{10} \mathrm{H}_{14} \mathrm{O}$, and 1-cyanoadamantane, $\mathrm{C}_{10} \mathrm{H}_{15} \mathrm{CN}$.

2-adamantaone [2-one-tricycle[3,3,1,1]-decane] is obtained from adamantane by substitution of two hydrogen atoms with oxygen. Below the melting point $\left(T_{m}=529 \mathrm{~K}\right)$ this compound has an orientationally disordered hightemperature phase with face-centered-cubic (Fm3m) symmetry, where the molecules exhibit reorientational motions of the dipolar axis along the six four-fold $<001>$ directions. The low-temperature ordered phase has been recently determined and it is orthorhombic with $C m c 2_{1}$ space group. Additionally a metastable monoclinic phase of $P 2_{1} / c$ space group was found just below the transition temperature [7]. This monoclinic phase displays an intrinsic disorder related with the site occupancy of oxygen atoms along three different sites. Such a disorder gives rise to a glass transition at ca. $130 \mathrm{~K}$.

Compared to 2-adamantanone, in 1-cyanoadamantane [1-cyano-tricylo[3,3,1,1]decane] the oxygen atom is replaced by the cyano group. Between the melting temperature $T_{m}=458 \mathrm{~K}$ and $T \approx 280 \mathrm{~K}$ the plastic phase exists, with the same symmetry as in the former compound, namely, fcc symmetry with space group Fm3m. At room temperature the molecular long axes are randomly distributed among the six orientations corresponding to the six crystallographic directions $<001>$. Below $280 \mathrm{~K}$ the orientational order appears as monoclinic phase with space group $C 2 / m$. But the glassy state is observable only below ca. $170 \mathrm{~K}$. From our previous examination we could conclude that more than one metastable state is present $[8,9]$.

Many calorimetric studies performed on these materials have marked the importance of the previous thermal history of the sample on the phase transition proper, which prompted us to examine this phenomenon $[4,10]$.

\section{Sample preparation}

2-adamantanone and 1-cyanoadamantane samples were purchased as powders from Sigma-Aldrich ${ }^{\circledR}$ with purity of 99 and 97\%, respectively, and were used without further purification. The primary form of the sample did not allow us to conduct any intended experiments and therefore needed to be transformed into a solids of a certain size. The final cylindrical shape of the sample was obtained by mechanical pressing at a pressure of approximately $1500 \mathrm{~kg} / \mathrm{cm}^{2}$. As far as thermal conductivity measurements are concerned, the whole compressed sample was used, whereas for heat capacity measurements a sufficient amount of sample was cut off for a better accuracy.

\section{Experiment}

In this work the thermal conductivity measurements were performed using the uniaxial stationary heat flux method in the specially designed bath cryostat [11]. This technique allows us to measure thermal conductivities to a great accuracy as a function of temperature. The stationary conditions are obtained by applying a fixed amount of power to the upper end of the sample by means of a resistive heater, while the other end is anchored to the so-called cold finger. The experimental setup is shown schematically in Fig. 1. The heater is glued upon by adhesive BF-2 and so is the sample to the cryostat. The temperature inside the experimental chamber was controlled with two Cernox ${ }^{\mathrm{TM}}$ thermometers, but the gradient of the temperature along the sample was measured with constantan-manganese thermocouples. The data collection process is carried out in high vacuum conditions obtained by rotary and turbo-molecular pumping systems. In addition, to avoid parasitic heat transfer, several isolating screens were distributed inside the cryostat maintaining the temperature distribution of the sample. In order to obtain the thermal conductivity the temperature drop along the sample was recorded and the formula below was used

$$
\kappa=\frac{P_{S}}{\Delta T_{S}} \frac{l}{A},
$$

where $l$ is the distance between thermocouples, $A$ is the cross-section of the sample, $P_{S}$ is the input power, $\Delta T_{S}$ is the resulting temperature difference.

The heat capacity measurements were carried out using the physical property measurement system (PPMS) from Quantum Design Inc in heat capacity option. The main

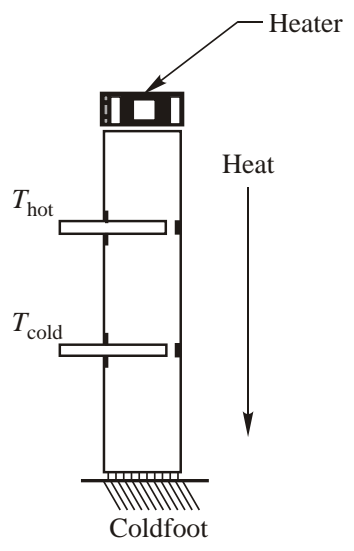

Fig. 1. Thermal connections of an idealized sample. 


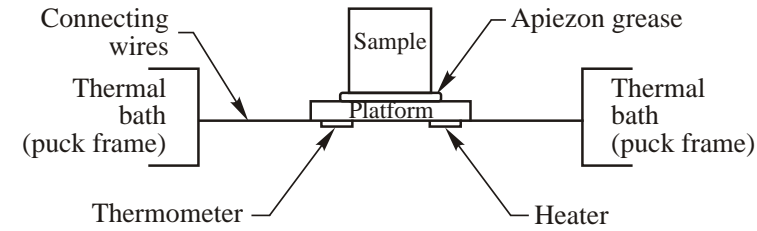

Fig. 2. Draft of the thermal connection between the sample and the sample platform in PPMS heat capacity option [12].

feature of the thermal relaxation method used by PPMS is to measure the heat capacity at constant pressure and to control the heat added and removed from a sample while simultaneously monitoring the resulting change in temperature [12]. After each cycle the system fits the temperature response of the sample platform with a thermal analysis model that comprises both the thermal relaxation of the sample platform to the bath (puck) temperature and the relaxation between the sample platform and the sample. The technique of measurement is simple, a certain amount of heat $P(t)$ is applied at constant power for a fixed time period, which is followed by cooling process of the same time (power off). For samples with imperfect thermal attachment to the sample platform the value of the heat capacity is determined by the resulting temperature difference according to the two tau model [13]:

$$
\begin{gathered}
C_{\text {platform }} \frac{d T_{p}}{d t}=P(t)-K_{w}\left[T_{p}(t)-T_{b}\right]+K_{g}\left[T_{p}(t)-T_{s}(t)\right], \\
C_{\text {sample }} \frac{d T_{s}}{d t}=-K_{g}\left[T_{p}(t)-T_{s}(t)\right]
\end{gathered}
$$

where $C_{\text {platform }}$ is the heat capacity of the sample platform, $C_{\text {sample }}$ is the heat capacity of the sample, $K_{w}$ is the thermal conductance of the wires and $K_{g}$ is the grease-related thermal conductance between the sample and the platform. The respective temperatures of the platform and sample are $T_{p}(t)$ and $T_{s}(t)$.

The draft of thermal connections in PPMS heat capacity option is shown of Fig. 2. During measurements the sample is located on the platform with little grease coating to improve the thermal contact. These conditions necessitate extraction of the heat capacity of apiezon grease from the gross results and, hence, performing a preliminary measurements of the grease coating alone. The electrical connection between the system and the sample is provided with the platform heater and thermometer located on the bottom side of the sample platform. The high-vacuum conditions were ensured by the PPMS turbo pump or the cryopump high-vacuum option. Those circumstances ensured sufficient thermal equilibrium necessary for the measurements.

\section{Results}

The results of thermal conductivity measurements of 2-adamantanone samples obtained with different cooling rates are presented in Fig. 3 in a double logarithm scale.

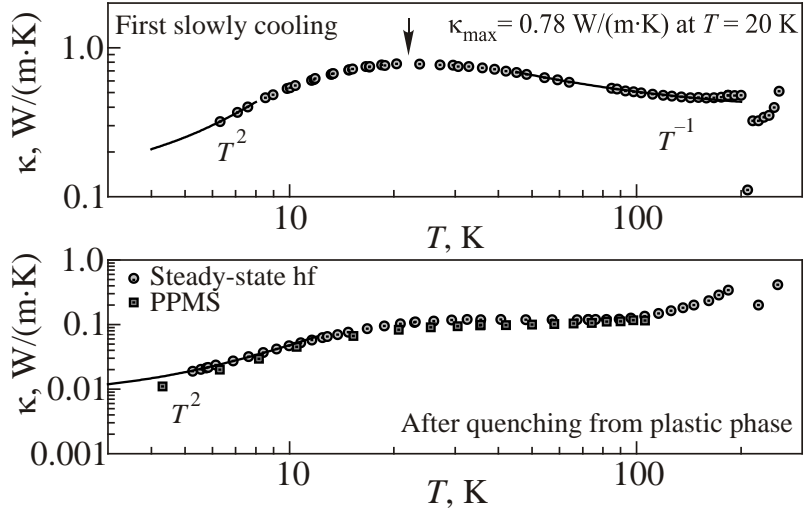

Fig. 3. Thermal conductivity $\kappa$ of 2-adamantanone after different thermal treatments.

The upper panel shows the values of thermal conductivity after slow cooling at a rate of about $1.5 \mathrm{~K} / \mathrm{min}$, i.e., for the low-temperature monoclinic phase. The $\kappa(T)$ curve resembles the characteristic behavior of ordered molecular crystals [14]. However, at low temperatures the thermal conductivity seems to follow the $T^{2}$ dependence instead of the typical $T^{3}$. The reason for that could be the fact that samples are not "perfect crystals". They may contain a substantial amount of point defects or dislocations that decrease the exponent in the temperature dependency and also make the following maximum of thermal conductivity shift to higher temperatures. The highest value of $0.78 \mathrm{~W} /(\mathrm{m} \cdot \mathrm{K})$ is reached at ca. $20 \mathrm{~K}$. The Umklapp processes limit the conductivity and thus contribute to the emergence of the maximum in $\kappa(T)$, dominate the phonon scattering mechanisms at higher temperatures. Therefore, in this temperature domain we can approximate our data with the Leibried-Schlömann 1/T law [15]. However, not the entire temperature range up to the phase transition point at about $202 \mathrm{~K}$ could be approximated with the same law. We must consider the presence of another mechanism responsible for the deviation. The lower panel shows the results obtained after quenching the sample. From our

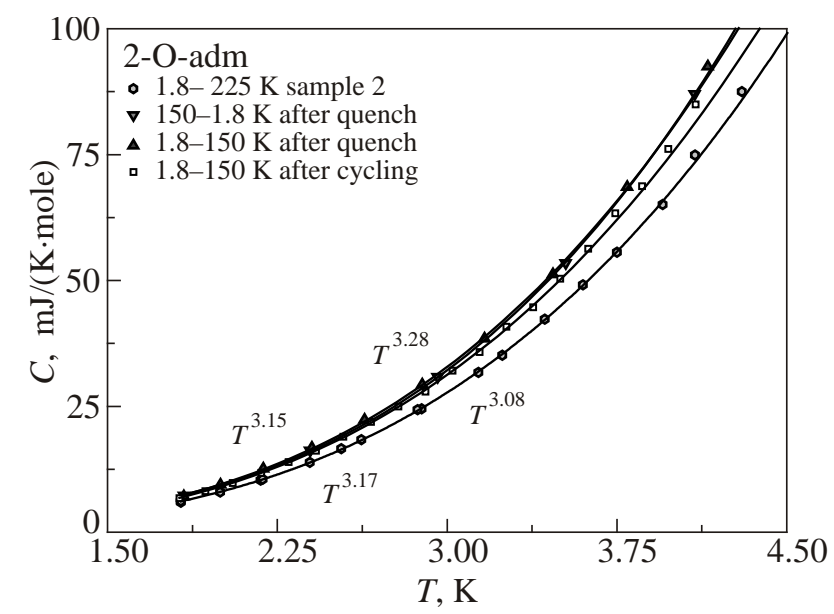

Fig. 4. Molar heat capacity $C$ of 2-adamantanone samples in the low-temperature region. 

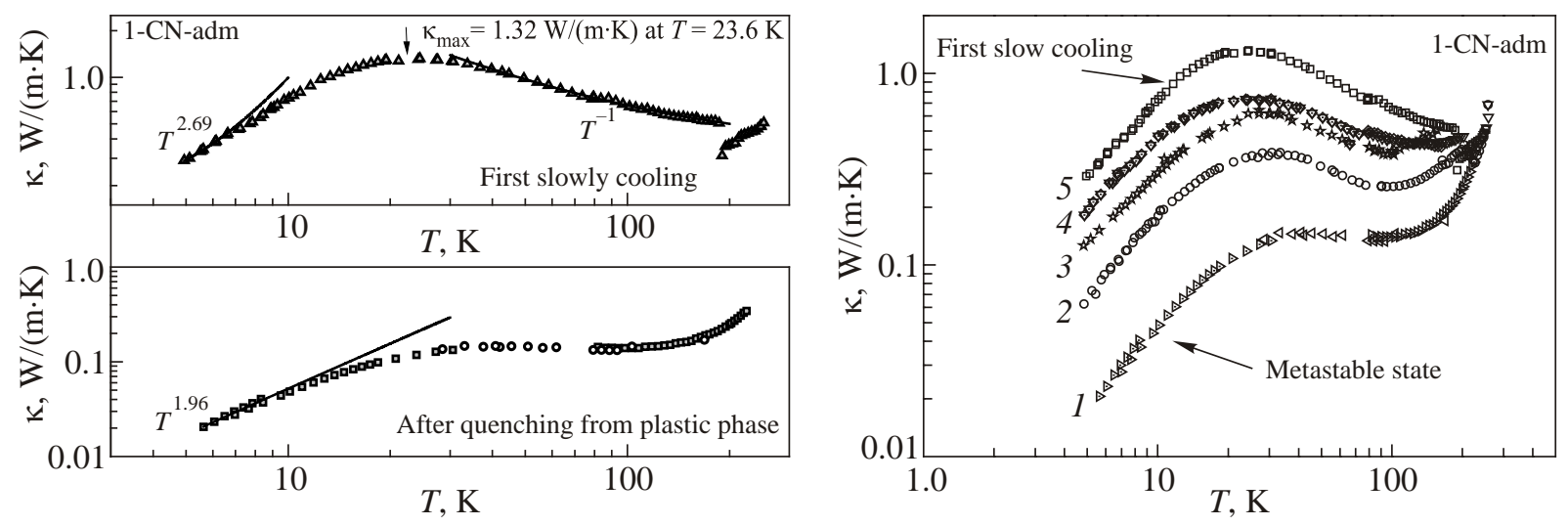

Fig. 5. Thermal conductivity $\kappa$ results for 1-cyanoadamantane samples for different cooling rates: after the first cooling by $1.5 \mathrm{~K} / \mathrm{min}$ from room temperature to $2 \mathrm{~K}$ (1); the second (2), third (3), fourth (4), and fifth (5) cooling by $0.5 \mathrm{~K} / \mathrm{min}$ from 250 to $2 \mathrm{~K}$.

measurement we concluded that the rate of cooling of about $7 \mathrm{~K} / \mathrm{min}$ is sufficient to freeze the metastable state $[8,9]$ and to observe an atypical glass-like behavior of our material. At the lowest temperatures, $\kappa(T)$ follows the $T^{2}$ dependence, expected for amorphous materials [16]. The standard consequence is the appearance of a plateau, but in our compound it emerges just above $20 \mathrm{~K}$.

Figure 4 presents results for the molar heat capacity measurement in 2-adamantaone. Although the experiment was carried out in the temperature range $1.8-300 \mathrm{~K}$, for discussion purposes we present the results in a very narrow temperature range. These data exhibit no essential distinction compared to the curves obtained for a different thermal history. Generally, the heat capacity at low temperatures should follow the $T^{3}$ dependence according to the Debye law [17]. Even though one could expect to witness some differences after quenching the metastable state $[8,9]$ (with the cooling rate of $10 \mathrm{~K} / \mathrm{min}$ ), there was no significant influence on the structure of our specimen at low temperatures. The slight change of the temperature dependence from $T^{3.08}$ to $T^{3.28}$ reflects only the natural scatter between particular samples.

For 1-cyanoadamantane samples similar measurements were also performed. The results of thermal conductivity

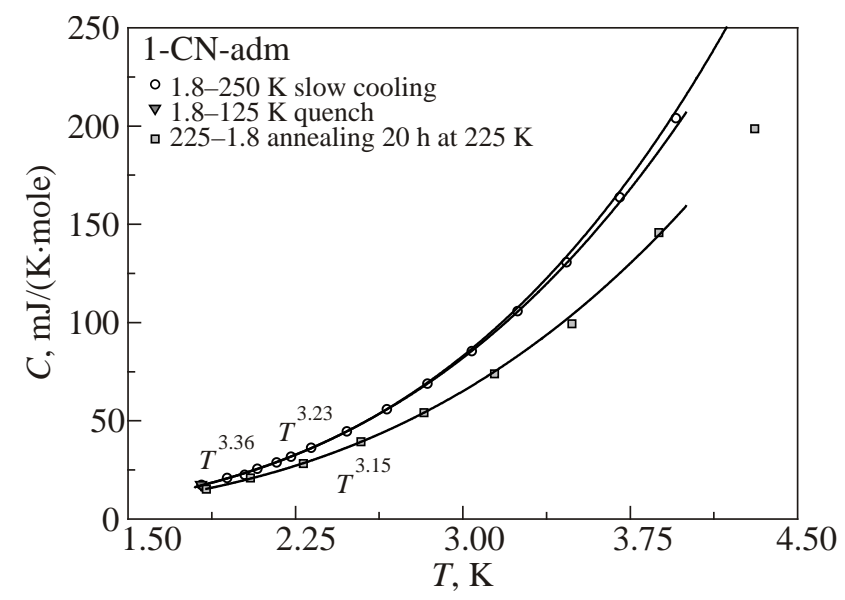

Fig. 6. Molar heat capacity $C$ of 1-cyanoadamantane in low-temperature region. are summarized in Fig. 5. During data collection we observed a few metastable states, some of them are shown in the right hand inset, the left one resembling the measuring procedure of the preceding material. After cooling slowly the sample we obtained the highest conductivity values. The maximum appears at about $24 \mathrm{~K}$ yielding $1.32 \mathrm{~W} /(\mathrm{m} \cdot \mathrm{K})$ for the thermal conductivity. The low-temperature dependence is closer to $T^{3}$ as for 2-adamantanone, which suggests that crystallization in 1-cyanoadamantane does not give rise to a high number of nanocrystals in which a strong phonon scattering takes place. The results for the quenched sample are shown in the lower left panel. Here also, the recorded data resembles the temperature response of amorphous solids: we observe a disordered crystal behavior with a small anomaly. Above the plateau the thermal conductivity increases, implying that the reported ferroelectric structure of 1-cyanoadamantane has a predominant contribution [8].

Similar heat capacity measurements were carried out for 1-cyanoadamantane samples; the results are presented on Fig. 6. Except for the differentiating of the cooling rate of the specimen - repeating the cooling procedure of the 2-adamantanone, additionally guided by the work of Yamamuro et al. [18] we submitted the samples to $20 \mathrm{~h}$ annealing just below the transition point. As an outcome we manage to measure the heat capacity of a completely ordered state of 1-cyanodamantane. The tendency of having no significant distinction at low temperatures observed in 2-adamantaone in this case remained. For both samples we could see a difference in results of thermal conductivity measurement after changing the rate of cooling, but when we repeated the cooling procedure for the heat capacity experiments, there was no change in the response of the samples. This kind of behavior is unusual as for amorphous solids [19].

\section{Conclusions}

By analyzing the thermal conductivity and the heat capacity of 2-adamantanone and 1-cyanoadamantane we could make some preliminary assumptions about thermal transport mechanisms involved in these materials. The most 
striking conclusion is that thermal treatment, in our case specifically the cooling rate, has an significant impact on the behavior of the materials. For both: 2-adamantanone and 1-cyanoadamantane we could investigate the lowtemperature ordered phase as well as the frozen-in metastable state. The singularity observed in our heat capacity measurements, that there was no pursuit of the system response observed in thermal conductivity results deriving from particular cooling processes, should be carefully addressed in our next works. There are still a lot of experiments left to be carried out and we hope they will bring us closer to the understanding of the processes present in these plastic crystals.

\section{Acknowledgments}

The work was supported by Wroclaw Research Centre EIT+ within the project "The Application of Nanotechnology in Advanced Materials” — NanoMat (POIG.01.01.0202-002/08) co-financed by the European Regional Development Fund (operational Programme Innovative Economy, 1.1.2).

1. J. Timmermans, J. Chim. Phys. 35, 331 (1938).

2. R. Brand, P. Lukenheimer, and A. Loidl, J. Chem. Phys. 116, 10386 (2002).

3. Th. Bauer, M. Köhler, P. Lukenheimer, A. Loidl, and C.A. Angell, J. Chem. Phys. 133, 144509 (2010).

4. I.S. Butler, H.B.R. Cole, D.F.R. Gilson, P.D. Harvey, and J.D. McFarlane, J. Chem. Soc. Faraday Trans. 82, 535 (1986).
5. R. Böhmer, G. Diezemann, G. Hinze, and E. Rössler, Prog. NMR Spectr. 39, 191 (2001).

6. K. Kobashi, T. Kyomen, and M. Oguni, J. Phys. Chem. Solids, 59, 667 (1998).

7. P. Negrier, M. Bario, M. Romanini, J.Ll. Tamarit, D. Mondeig, A.I. Krivchikov, L. Kepinski, A. Jezowski, and D. Szewczyk, Cryst. Growth Des. 14, 2626 (2014).

8. R. Fabiański, L. Firlej, and B. Kuchta, J. Chem. Phys. 116, 2310356 (2002).

9. B. Kuchta, M. Descamps, and F. Affouard, J. Chem. Phys. 109, 166753 (1998).

10. L. Bistricić, G. Baranović, and V. Volovšek, J. Mol. Struc. 482, 661 (1999).

11. T. Zakrzewski and H. Misiorek, Polish Techn. Rev. 7, 13 (1976).

12. Quantum Design (2000). Physical Property Measurement System. User’s Manual. Quantum Design, 11578 Sorrento Valley Rd. San Diego, CA 92121-1311, USA.

13. J.C. Lashley, J. Singleton, A. Migliori, J.B. Betts, R.A. Fisher, J.L. Smith, and R.J. McQueeney, Cryogenics, 43, 369 (2003).

14. R. Berman, Thermal Conduction in Solids, Oxford University Press (1976).

15. G. Leibfried and E. Schlömann, Nach. Akad. Wiss. Gottingen, Math. Phys. Klasse 4, 71 (1954).

16. R.O. Pohl, X. Liu, and E.J. Thompson, Rev. Mod. Phys. 74, 991 (2002).

17. P. Debye, Ann Phys. 344, 789 (1912).

18. O. Yamamuro, M. Ishikawa, I. Kishimoto, J.-J. Pinvidic, and T. Matsuo, J. Phys. Soc. Jpn. 68, 2969 (1999).

19. M.P. Zaitlin and A.C. Anderson, Phys. Rev. B 12, 4475 (1975). 